\title{
Parameatal cyst in a 4-year-old boy
}

\author{
Gérard Lorette - Marie-Christine Machet • \\ Annabel Maruani
}

Received: 13 June 2013 / Accepted: 10 July 2013 / Published online: 31 July 2013

(C) Springer-Verlag Berlin Heidelberg 2013

\begin{abstract}
A 4-year-old boy presented a translucent cyst on the left lateral side of the urethral meatus. The lesion was excised. Histological examination showed features of a cystic lesion within the dermis surrounded by a thin epithelium of columnar cells with a round apical pole. Immunohistochemical staining was positive for pan-cytokeratins (AE-1/AE-3) and negative for actin. These findings led to a diagnosis of urethral parameatal cyst. In conclusion: this entity is a rare congenital malformation and consists of a parameatal benign cyst occurring in prepubertal males. Treatment consists of complete resection of the cyst.
\end{abstract}

Keywords Parameatal cyst $\cdot$ Malformation

A 4-year-old boy presented a translucent cyst on the left lateral side of the urethral meatus (Fig. 1), which had appeared 2 years before. He had no history of urinary disorders and no urinary symptoms. Physical examination was otherwise normal. $\mathrm{He}$ received no treatment.

The clinical diagnosis was dysembryoplastic cyst. There was no other diagnosis to discuss. Indeed, the persistence of the bullae does not allow the diagnosis of autoimmune bullous disease, and Herpes vesicles would be grouped transient and painful. The lesion was excised. Histological examination showed features of a cystic lesion within the dermis surrounded by a thin epithelium of columnar cells with a round apical pole. Immunohistochemical staining was positive for

G. Lorette $\cdot$ A. Maruani

Department of Dermatology, CHU Tours, Université François

Rabelais Tours, Tours, France

M.-C. Machet

Department of Pathology, Université François Rabelais Tours,

Tours, France

G. Lorette $(\bowtie)$

Department of Dermatology, Trousseau Hospital, CHRU Tours, Avenue de la République, 37044 Tours Cedex 9, France

e-mail: lorette@med.univ-tours.fr pan-cytokeratins (AE-1/AE-3) and negative for actin. These findings led to a diagnosis of urethral parameatal cyst.

This entity is a rare congenital malformation and consists of a parameatal benign cyst occurring in prepubertal males. Histologically, the cyst can contain a single type of epithelium or a combination of transitional, cuboidal and/or columnar epithelia. Although the mechanism is poorly understood, immunostaining and scanning electron microscopy findings suggest that the cyst developed from the epithelium of the urethra [2].

Treatment consists of complete resection of the cyst, for minimal risk of recurrence. Aspiration of the content of the cyst or resection of the roof may induce recurrence [1].

Conflict of interest None

\section{References}

1. Ouaran M, Tan MO, Camtosum A, Irkilata L, Erdem O, Bozkirli I (2006) Parameatal anomaly. Int Urol Nephrol 38:273-274

2. Yamada N, Yoshida Y, Ito A, Monno S, Yamamoto O (2013) Threedimensional structure of a parameatal urethral cyst by scanning electron microscopy. Urology 81:1072-1074

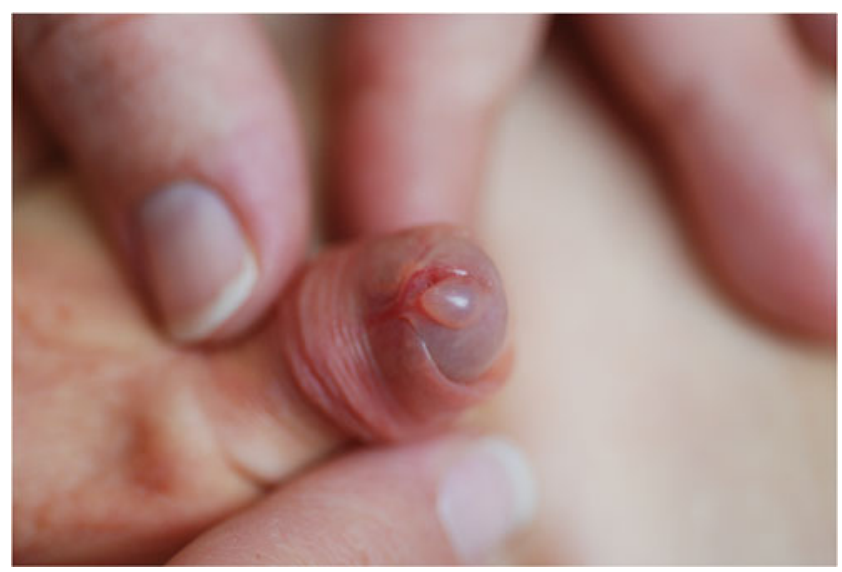

Fig. 1 Parameatal cyst in a 4-year-old boy 